\title{
Transcriptome analysis and molecular marker discovery in Solanum incanum and S. aethiopicum, two close relatives of the common eggplant (Solanum melongena) with interest for breeding
}

\author{
P. Gramazio*, J. Blanca, P. Ziarsolo, F. J. Herraiz, M. Plazas, J. Prohens and S. Vilanova
}

\begin{abstract}
Background: Solanum incanum is a close wild relative of S. melongena with high contents of bioactive phenolics and drought tolerance. S. aethiopicum is a cultivated African eggplant cross-compatible with S. melongena. Despite their great interest in S. melongena breeding programs, the genomic resources for these species are scarce.

Results: RNA-Seq was performed with NGS from pooled RNA of young leaf, floral bud and young fruit tissues, generating more than one hundred millions raw reads per species. The transcriptomes were assembled in 83,905 unigenes for S. incanum and in 87,084 unigenes for S. aethiopicum with an average length of 696 and 722 bp, respectively. The unigenes were structurally and functionally annotated based on comparison with public databases by using bioinformatic tools. The single nucleotide variant calling analysis (SNPs and INDELS) was performed by mapping our S. incanum and S. aethiopicum reads, as well as reads from S. melongena and S. torvum available on NCBI database (National Center for Biotechnology Information), against the eggplant genome. Both intraspecific and interspecific polymorphisms were identified and subsets of molecular markers were created for all species combinations. 36 SNVs were selected for validation in the S. incanum and S. aethiopicum accessions and $96 \%$ were correctly amplified confirming the polymorphisms. In addition, 976 and 1,278 SSRs were identified in S. incanum and S. aethiopicum transcriptomes respectively, and a set of them were validated.

Conclusions: This work provides a broad insight into gene sequences and allelic variation in S. incanum and S. aethiopicum. This work is a first step toward better understanding of target genes involved in metabolic pathways relevant for eggplant breeding. The molecular markers detected in this study could be used across all the eggplant genepool, which is of interest for breeding programs as well as to perform marker-trait association and QTL analysis studies.
\end{abstract}

Keywords: Solanum incanum, S. aethiopicum, Eggplant genepool, De novo transcriptome, Gene annotation, Molecular marker discovery

\footnotetext{
* Correspondence: piegra@upv.es

Instituto de Conservación y Mejora de la Agrodiversidad Valenciana,

Universitat Politècnica de València, Camino de Vera 14, 46022 Valencia, Spain
} 


\section{Background}

The global production of eggplants has considerably increased, especially in Asia and Africa, rising from 29 to 49 millions of tons in the last decade [1]. Despite its economic importance, compared to other major vegetable crops, few efforts have been made to use related species for the genetic enhancement of common eggplant (Solanum melongena L.). In this respect, resistance and tolerance to biotic and abiotic stresses, as well as high levels of bioactive compounds have been found in S. melongena relatives [2-4], but they have not been widely used in breeding programs.

Although S. melongena, which was domesticated in Southeast Asia [5], is by far the economically most important cultivated eggplant, there are two other cultivated eggplant species of African origin, S. aethiopicum L. and S. macrocarpon L., which are major vegetable crops [6, 7]. The three species, together with a large number of wild species from the eggplant clade and the anguivi clade of Solanum subgenus Leptostemonum [8], form part of the same genepool. Most of these species can be successfully hybridized with S. melongena [4]. Among them the wild S. incanum L. and the cultivated $S$. aethiopicum have been regarded as valuable sources of variation for $S$. melongena breeding [3, 10-13].

Solanum incanum, which has been regarded for a time as the wild ancestor of S. melongena [14], is naturally distributed in desert and dryland areas in a broad area between northern Africa and the Middle East of Pakistan [15]. S. incanum is considered a powerful source of bioactive phenolics, mainly chlorogenic acid (5-O-caffeoylquinic acid) and to lesser extent $\mathrm{N}-(\mathrm{E})$-caffeoylputrescine, 3-O-malonyl-5-O-(E)-caffeoylquinic acid and 5-O-malonyl4-O-(E)-caffeoylquinic acid [12], showing a content several times higher than that of cultivated eggplants [12, 13, 16$]$. Moreover, $S$. incanum presents resistance at some fungal diseases, like Fusarium oxysporum and Phomopsis vexans and tolerance to abiotic stresses such as drought $[2,14,17]$. Fully fertile interspecific hybrids have been obtained between $S$. incanum and $S$. melongena with a regular meiosis $[12,14,18,19]$, as well as a backcross population to $S$. melongena that has allowed the development of an interspecific genetic linkage map [20]. S. aethiopicum is the second most important cultivated eggplant, and its cultivation is widespread in Africa, mainly in the west and central part, as well as in some parts of Caribbean, Brazil and south Italy [7, 21, 22]. Generally, this species is divided in four cultivar groups, namely Aculeatum, Gilo, Kumba, and Shum [23], with the Gilo group, used for its edible oval to rounded fruits, the most important group in the $S$. aethiopicum complex [3, 22, 23]. S. aethiopicum is of interest for $S$. melongena breeding as resistance to fungi (Fusarium oxysporum, F. solani, Pythium vexans, Phytophthora parasitica), bacteria (Ralstonia solanacearum), insect (Leucinodes orbonalis) as well as root-knot nematodes (Meloidogyne incognita) has been found in different materials of this species [9, 24-26]. Although different degrees of fertility have been found in interspecific hybrids between $S$. melongena and S. aethiopicum, backcrosses to $S$. melongena and introgression materials have been obtained [9-11]. As S. aethiopicum is a cultivated species, it does not present undesirable traits characteristic of wild species (e.g., prickliness, small fruit size, high content in saponins and glycoalkaloids, seed dormancy, etc.) that have to be removed in breeding programs. Also interspecific hybrids between $S$. melongena and S. aethiopicum are highly vigorous and of interest for being used as rootstocks of S. melongena [26].

Despite the importance of eggplants for security food for millions of people, genomics studies in this group have been limited. Only S. melongena has received some attention, with several intraspecific and interspecific genetic maps [20, 27-30], collections of molecular markers $[29,31,32]$ a set of 16000 unigenes [27], a de novo transcriptome assembly [33], and a draft genome sequence [34] being available. Genomic resources in S. melongena relatives are generally scarce. In this respect, while for $S$. melongena there are 126,715 DNA and RNA sequences deposited in NCBI [35] nucleotide database (on September 2015), of which 100,389 correspond to ESTs (expressed sequence tag) sequences, only 68 sequences are available from S. aethiopicum, none of which is an ESTs. In S. incanum, a transcriptome assembly was released recently (GAYS 00000000.1), but it is still unpublished and no analyses have been released up to now.

The only exception concerns S. torvum Swartz, with 133,602 DNA and RNA sequences, of which 28,745 are ESTs. Solanum torvum, also known as turkey berry, is used as a vegetable and medicinal plant [36-38]. Also $S$. torvum shows resistance to many plant and soil-borne disease, such as F. oxysporum, Verticillium dahliae, $P$. parasitica, R. solanacearum, and Meloidogyne spp. [2, 39, 40]. Many efforts have been done to transfer these resistances through different biotechnological approaches, due to the high sterility of hybrids obtained via conventional crosses [41-45]. However, at present, the main use of S. torvum is as a rootstock for eggplant [46-48].

Yang et al. [33] sequenced simultaneously the transcriptomes of $S$. torvum and S. melongena, providing valuable sets of unigenes and detailed information about the two species. However, this study did not include the discovery of molecular markers, which could have been of great assistance in the breeding programs within and between each species.

The aims of the present study are building two transcriptomes from S. incanum and S. aethiopicum through the generation of ESTs using RNA-Seq, providing genomic tools in these relatives of $S$. melongena. This will 
be the starting point for gene discovery, splicing patterns and other post-transcriptional modifications, as well as expression levels of transcripts during development and under different conditions. Furthermore, the trimmed transcripts of S. aethiopicum and S. incanum and the transcripts of $S$. melongena and S. torvum, downloaded from NCBI database [35], were mapped against the eggplant genome to discover the molecular variations within and between species in order to create large subsets of markers directly applicable in breeding programs along to the eggplant genepool. All this information will contribute to the utilization of these species for S. melongena breeding, as well as to the enhancement of the cultivated, but neglected, S. aethiopicum.

\section{Results and discussion}

\section{Illumina paired-end sequencing and EST assembly}

The new generation of high-throughput sequencing platforms and the improved algorithms for de novo transcriptome assembly has allowed the availability of transcriptomes even in non-model organisms without a reference genome [49]. In our case, in order to build a transcriptome of S. incanum and S. aethiopicum, mixed RNA from young leaf, floral bud and young fruit, was used in order to increase the heterogeneity and diversity of the transcripts. Two different libraries were constructed, one per species, and subsequently sequenced in a HiSeq 2000 sequencer (Illumina).

A total of $105,625,594$ and $114,162,500$ raw reads were obtained from S. incanum and S. aethiopicum respectively (Table 1), which have been deposited in the NCBI Sequence Read Archive (Bioproject SRP063088) [35]. After the filtering and trimming process, removing adapters and low Phred quality sequences, 91,579,142 and 99,012,712 high-quality sequences were obtained for S. incanum and $S$. aethiopicum. The trimmed reads were assembled into transcriptomes using Trinity software [50], generating 108,322 transcripts for S. incanum and 106,660 for S. aethiopicum (Additional file 1). Subsequently, in order to test the overall assembly quality, the clean reads were mapped against the transcriptomes using Bwa [51], which is an ultrafast and memory-efficient mapper particularly good at aligning reads between 50 to $100 \mathrm{bp}$. The large number of reads properly mapped, specifically $94.3 \%$ for S. incanum and $95.9 \%$ for S. aethiopicum, confirmed the high quality of Trinity assembly. The total length of assembled transcripts for S. incanum and S. aethiopicum was about 102 and $92 \mathrm{Mbp}$ with an average length of 946 and $868 \mathrm{bp}$, respectively. In recent years the assemblies have been improved progressively as a result of the advances in sequencing platforms, especially Illumina [52-54].

Trinity software determines splice variants (isoforms) and distinguishes transcripts from recently duplicated and identified allelic variants [50]. To obtain a set of
Table 1 Statistics of S. incanum and S. aethiopicum assembled transcripts and unigenes, using Trinity software

\begin{tabular}{lll}
\hline & S. incanum & S. aethiopicum \\
\hline Raw reads & $105,625,594$ & $114,162,500$ \\
Sequence lenght & 101 & 101 \\
Mean sequence quality (Phred Score) & 36.03 & 36.02 \\
\%CG & 43 & 43 \\
High-quality reads & $91,579,142$ & $99,012,712$ \\
Sequence lenght & $70-101$ & $70-101$ \\
Mean sequence quality (Phred Score) & 36.88 & 36.87 \\
\%CG & 42 & 42 \\
Transcript & 108,322 & 106,660 \\
Max lenght & 12,202 & 12,179 \\
Avarage lenght & 946 & 868 \\
N50 & 1,693 & 1,455 \\
Total residues & $102,496,435$ & $92,629,886$ \\
Unigenes & 83,905 & 87,084 \\
Max lenght & 12,181 & 12,159 \\
Avarage lenght & 696 & 722 \\
N50 & 1,153 & 1,139 \\
Total residues & $58,447,674$ & $62,899,378$ \\
\hline
\end{tabular}

Data correspond to the results of RNA-Seq projects by HiSeq 2000 sequencer (Illumina) of total RNA from three tissues of S. incanum and S. aethiopicum and subsequent processing

single-copy gene locus (unigene), only the most expressed transcript from the isoforms of each locus was chosen, using the RSEM software (RNA-Seq by ExpectationMaximization) [55]. A total of 83,905 unigenes were identified in S. incanum and 87,084 in S. aethiopicum, showing that $22.5 \%$ and $18.3 \%$ of transcripts were splice variants respectively (see Additional file 2).

Even though the $60 \%$ of unigenes had between 201 and $500 \mathrm{bp}$, more than $20 \%$ of them were longer than 1 $\mathrm{kbp}$. The length distribution of unigenes is shown in Fig. 1. In order to obtain the physical position of the unigenes, a BlastN against the $S$. melongena genome was performed and the results are illustrated in the Circos plot in Fig. 2 and Additional file 3 [56]. The distribution of unigenes was uneven along the eggplant genome. Most unigenes clustered in areas which could correspond to the short and long arms of chromosomes, while a lower unigene density was observed in regions which could correspond to the centromere and pericentromeric regions, based on the observation of Doganlar et al. [28]. This gene distribution is similar to the observed in other species $[57,58]$. Our sets of unigenes are much higher than those obtained for S. melongena and $S$. torvum. S. melongena unigenes set consisted of 16,245 [59] and 34,174 unigenes [33], while in Solanum torvum 38,185 unigenes were obtained [33]. A deeper sequencing and better coverage were probably the reasons of 


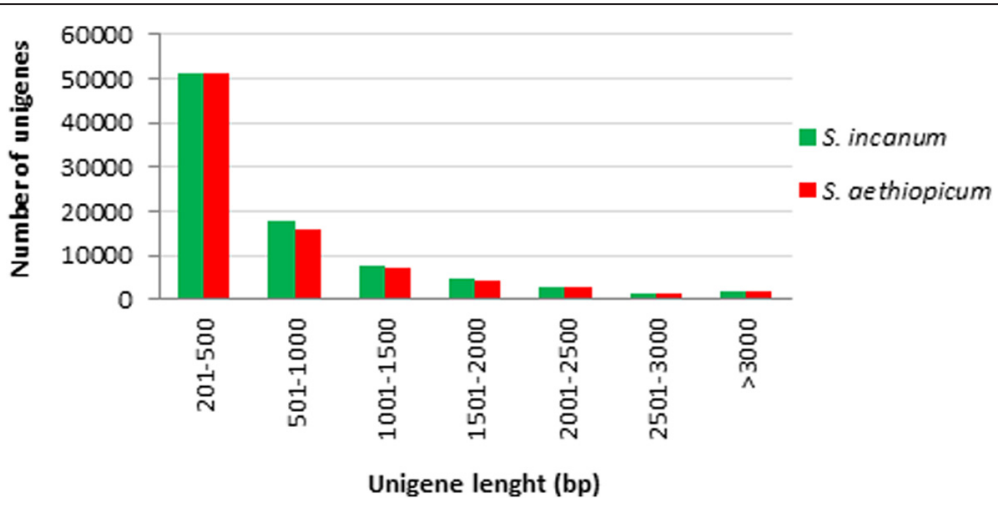

Fig. 1 Length distribution (bp) of S. incanum and S. aethiopicum unigenes

the greatest number of our S. incanum and S. aethiopicum datasets unigenes. In fact the number of our total residues was 58,447,674 for S. incanum and 62,899,378 for $S$. aethiopicum, higher than those obtained by Fukuoka et al. [59] and Yang et al. [33] in S. melongena (50,438,137 and 27,771,410, respectively), and Yang et al. [33] in S. torvum $(30,868,727)$. These higher numbers of unigenes has been observed in other plant transcriptomes recently published [60-62] in which the number of raw reads obtained was quite large.

\section{Annotation of S. incanum and S. aethiopicum transcriptomes}

Transcriptome annotation provides insight into the structural, functions and biological processes of assembled unigenes [63]. The functional annotation was performed using the assembled unigenes as query in BlastX searches against the three major protein databases and tomato protein database in this sequential order: Swiss-Prot [64], ITAG2.4 [65], Arabidopsis proteins [66] and Uniref90 [67]. Moreover a BlastX analysis was performed against the non-redundant (NR) protein database of NCBI [35] and the GO terms (Gene Ontology) and EC number (Enzyme Commission) have been assigned through the Blast2GO software [68].

A total of 30,630 (36.5 \%) S. incanum and 34,231 (39.3\%) S. aethiopicum have shown at least one hit in the protein databases. Most of the unigenes $(57.5 \%$ for S. incanum and $57.3 \%$ for S. aethiopicum) were annotated using the manually reviewed Swiss-Prot database and ITAG 2.4 (34.9\% and $33.2 \%)$ and less using Uniref90 (7.3 \% and $9.3 \%)$ and Arabidopsis protein database $(0.3 \%$ and 0.2$)$. The unigenes annotated under different protein database are reported in Additional file 4.

Even though the percentage of unigenes annotated in the protein databases seems to be quite low, the total number, 30,630 for S. incanum and 34,231 for S. aethiopicum, is consistent with the number of protein-coding genes described in tomato $(34,727)$ [69] and in previous works in other plant species. For instance in pepper transcriptome (Capsicum annum L.) [70] 24,003 out of 31,196 unigenes were annotated in protein databases as well as 32,410 out of 68,132 unigenes in Oryza officinalis Wall. ex Watt [71] and 34,368 out of 82,036 unigenes in litchi (Litchi chinesis Sonn.) [72]. Similarly, in S. melongena and S. torvum 28,016 and 29,845 unigenes were annotated, respectively [33].

A large portion of hitless unigenes were short sequences between 200 and $500 \mathrm{bp}$. This huge set of nonannotated short sequences has been observed in recent published transcriptomes, in which large amount of raw reads have been obtained $[61,73]$. Besides $3^{\prime}$ or $5^{\prime}$ untranslated regions (UTRs) and intron sequences from non-mature mRNAs, several authors have described that some of these sequences could be noncoding RNAs (ncRNAs) [74]. Unfortunately at the present the ncRNA is still in their early stages and just some mammalian entries were uploaded to IncRNA database [75]. Up to now, the only plant that has received some attention is Arabidopsis thaliana where 13,000 RNAs were found transcribed from intergenic regions [76, 77].

Gene ontology provides a systematic language in three key biological domains shared by all organisms: molecular function, biological process and cellular component to unify the representation of gene features across all species [39]. GO terms are structured as a graph and can be distributed in levels. Level 1 represents the most general categories and provides the most coverage, whereas higher levels provide more specific information and less coverage [39]. Briefly, level 1 is a general description of a process whereas higher levels provide a more specific description.

A total of 136,904 and 109,044 GO terms were assigned to 25,650 (30.5 \%) and 25,169 (28.9\%) unigenes in S. incanum and $S$. aethiopicum respectively. The GO annotation results are presented in Additional file 5. The GO terms per unigenes ranged from 1 to 92 for S. incanum and from 1 to 55 for S. aethiopicum, although most of the unigenes 


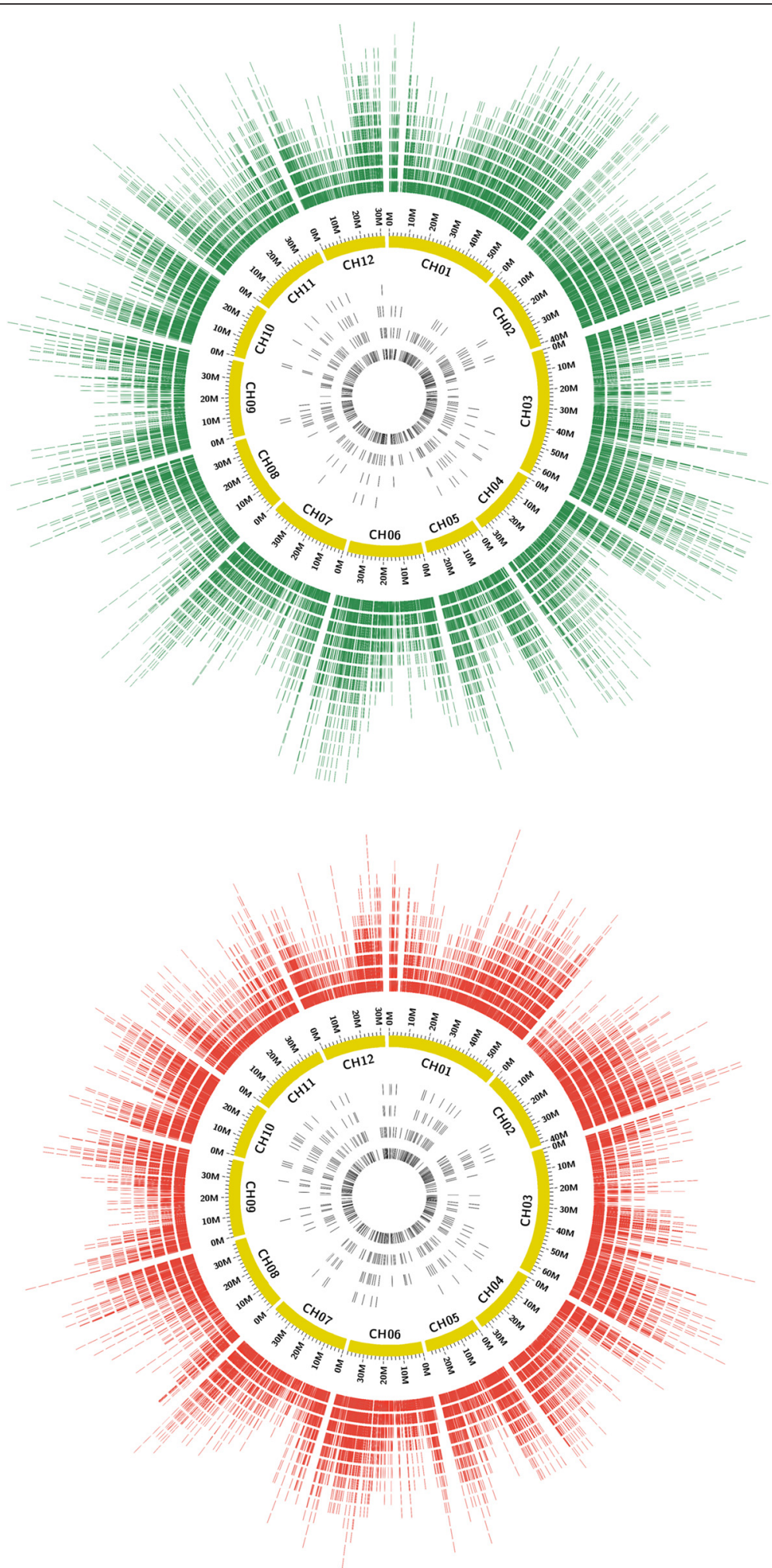

Fig. 2 Distribution of S. incanum (top) and S. aethiopicum (bottom) assembled unigenes and SSRs on S. melongena genome. In the outer histogram, depicted in green for S. incanum and in red for S. aethiopicum, are represented the assembled unigenes along the eggplant genome (yellow ring). Only unigenes which have given an E-value 0.0 via BlastN search were shown. In the inner histograms, in black, the distribution of the SSRs detected in each species is represented 
have 1 to $10 \mathrm{GO}$ terms (Fig. 3). The unigenes were also annotated with EC number [78], which identifies the reactions they catalyze. EC numbers were assigned to 8,343 $(9.9 \%)$ and 14,524 (16.6\%) unigenes, varying from 1 to 9 per unigene, although almost $80 \%$ presented only one EC number (Fig. 3).

The majority of GO terms ( $44.6 \%$ for S. incanum and $47.4 \%$ for S. aethiopicum) were related to biological processes (Fig. 4). Most of them had a GO annotation level in the range of 4 to 10 . Biological processes such as oxidation-reduction and metabolic processes, protein phosphorylation and regulation of transcription are usually specific of tissues in a developmental stage [79]. Molecular functions have been assigned to $30.7 \%$ and $35.4 \%$ of ontologies, most of them showing a GO annotation level of 3 to 9 and being the binding activities the most represented. The remaining $24.7 \%$ and $17.3 \%$ of annotated unigenes have shown a cellular component GO term, mostly related to nucleus, plasma membrane, cytosol, as well as chloroplast and mitochondria. The distribution of GO level for this category is quite uniform, with the exceptions of levels 5 and 8 .
The Kyoto Encyclopedia of Genes and Genomes (KEGG) is an integrated database resource, which links genomic data with functional information to standardize gene annotation [80]. Using Blast2GO software the annotated unigenes were blasted against the KEGG pathway database in order to dissect the molecular interaction among them. A total of 11,151 (13.2 \%) S. incanum unigenes were assigned to 146 KEGG biological pathways, involving 378 enzyme types and 879 EC numbers. Regarding S. aethiopicum 13,101 unigenes (15.0\%) were ascribed to 147 KEGG pathways, including 356 different enzymes and $821 \mathrm{EC}$ numbers. In $S$. incanum the three most enriched pathway were the biosynthesis of antibiotics, which included 713 unigenes (map01130), made mainly by phosphohexokinase (21 unigenes), dehydrogenase (18) and dehydrogenase (NAD+) (18) enzymes, Purine metabolism pathway (567 unigenes, map00230) was mostly represented by phosphatase (194 unigenes), adenylpyrophosphatase (130) and RNA polymerase (79) enzymes, and starch and sucrose metabolism pathway (516 unigenes, map map00500) was composed especially by pectin demethoxylase (85 unigenes), pectin depolymerase (64)

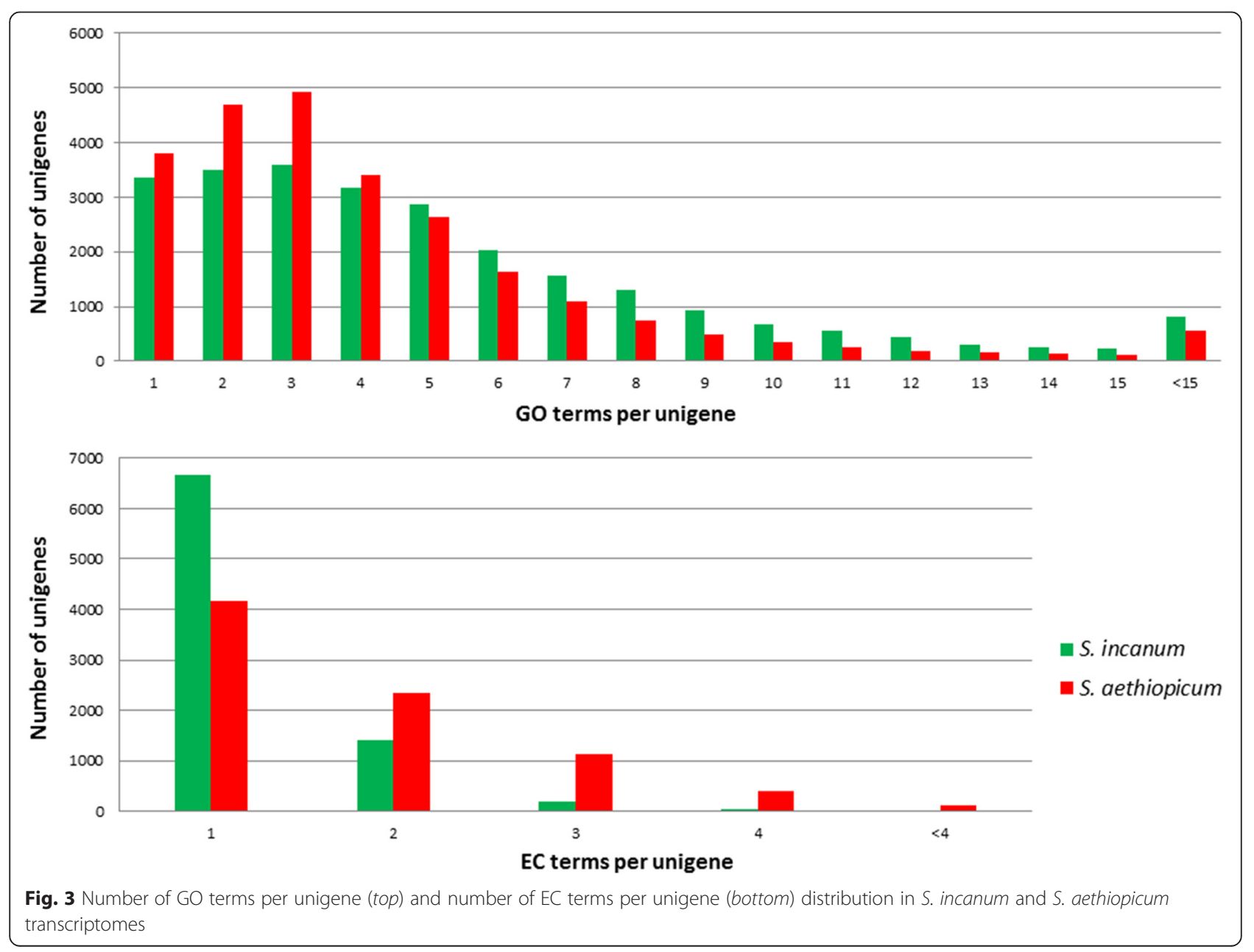




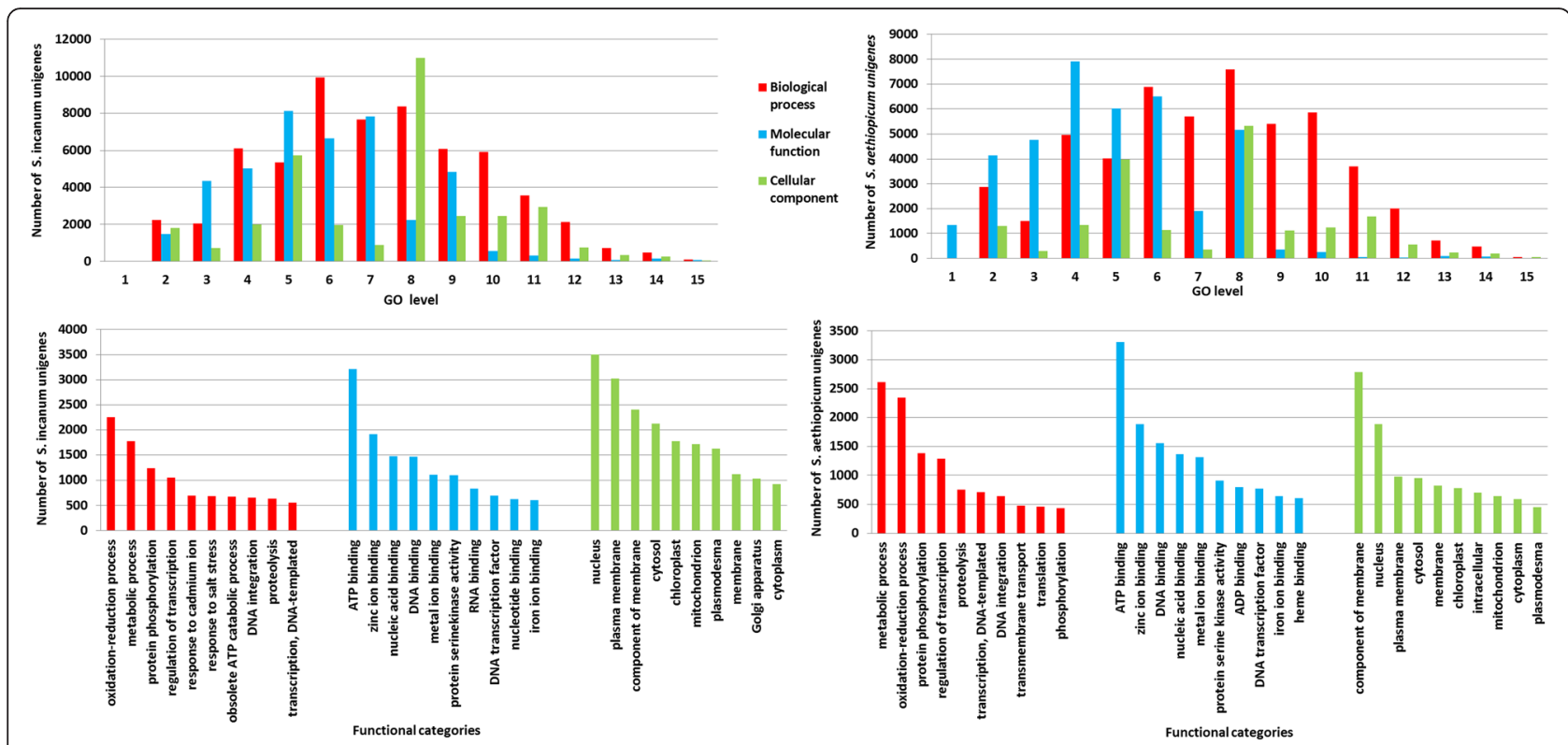

Fig. $4 \mathrm{GO}$ level distribution (top) and functional categories annotation (bottom) of the S. incanum (left) and S.aethiopicum (right) unigenes. On the top are shown the distributions of $\mathrm{GO}$ level in the following functional categories: biological process, molecular function and cellular component. On the bottom are represented the descriptions of the most abundant gene products for each functional category of GO ontologies: biological process, molecular function and cellular component

and UDP synthase (54) enzymes. In S. aethiopicum the most enriched pathway was purine metabolism (map00230), which encompasses 1404 unigenes, mostly constituted by phosphatase (1160 unigenes), adenylpyrophosphatase (630) and RNA polymerase enzymes (92), followed by thiamine metabolism pathway (1172, map 00730), made basically for phosphatase enzymes (1160 unigenes), and biosynthesis of antibiotics pathway (639 unigenes, map01130) including 168 enzyme types of which the most represented were dehydrogenase (20 unigenes), phosphohexokinase (18) and transaminase (16) enzymes. The biological pathways maps are reported in the Additional file 6 and the KEGG annotation results are compiled in the Additional file 7.

In order to establish a set of orthologs and gene model prediction, a best reciprocal Blast hits was performed with the tomato reference genome (version SL2.50) [69]. Up to now, the closest phylogenetically published genome of S. incanum and S. aethiopicum is the one of $S$. melongena [33]. Even though this first version has provided valuable information for eggplant breeding programs, it is just a draft, requiring a deeper and most complete sequencing. On the other hand, the S. lycopersicum genome is the most complete and accurate in genus Solanum. In the last version, SL2.50, the tomato genome scaffolds were re-ordered and re-oriented, and the gap sizes between scaffolds were set using FISH (Fluorescence in situ hybridization) and optical mapping. For that reason, apart from S. melongena, a BlastN was performed against $S$. lycopersicum. In the Additional file 8 the Blast hits results between the assembled unigenes and S. lycopersicum genome version SL2.50 are reported. A total of 16,388 (19.5\%) and 17,630 (20.2 \%) unigenes have presented orthologs with tomato reference genome in $S$. incanum and S. aethiopicum respectively (Additional file 9), while between the two transcriptomes 46,498 orthologs were found.

Regarding structural annotation, the ORF (Open reading frame) detection was performed using ESTScan software [81], which predicted 35,943 ORFs ( $42.8 \%$ of the total unigenes residues; Additional file 10) in S. incanum. The total number of nucleotides in S. incanum ORFs was $39,611,611$ (67,7 \% of total S. incanum unigenes assembled residues). In S. aethiopicum 40,353 ORFs were predicted (46.3\% of the total unigenes), which are constituted by $43,653,585$ nucleotides ( $69.4 \%$ of total S. aethiopicum unigenes assembled residues Additional file 10). Furthermore using est2genome [82] the intron regions were detected, providing valuable information about gene structure as well as in the task of primers design, avoiding regions in the intron edges proximity. In 12,368 S. incanum unigenes (14.7\% of the total unigenes) 59,501 introns were predicted, while 65,996 introns were detected in 13,661 S. aethiopicum unigenes (15.6\%) (Additional file 10). Both species have exhibited an average of 4.8 introns per unigene with a maximum of 49 for S. incanum and 56 for S. aethiopicum. The low percentage of introns detected in the unigenes is probably due to that the $60 \%$ of unigenes had between 201 and $500 \mathrm{bp}$ and for their small size did not contain any intron. 


\section{Molecular markers discovery and validation}

\section{Single nucleotide variations (SNVs)}

During the last decade Next-Generation Sequencing (NGS) techniques have allowed the development of large molecular marker collections with modest investments even in non-model species [83, 84]. These collections enable the location of thousands of single polymorphisms along the genome as well as the development of high-density genetic maps, arrays and genotyping assays [85-87].

Although genomic resources have been developed in eggplant (e.g., Barchi et al., [29]; Yang et al., [33] Hirakawa et al., [34]), few genomic information is available for closely related species. In the present study, large subsets of SNPs (Single Nucleotide Polymorphisms) and INDELs (insertion/deletions) have been identified to assist efficiently plant breeding projects and diversity studies. The SNP calling was performed for the species sequenced in the present study (S. incanum and S. aethiopicum) and for the two other species of the eggplant genepool (S. melongena and $S$. torvum), whose transcriptomes have already been sequenced [33]. The reads of the four transcriptomes have been mapped against the eggplant genome and SNPs detected using Freebayes SNP caller [88], as detailed in Methods section. The complete information of SNP calling is provided in Additional file 11. A set of $36 \mathrm{SNVs}$ (Single nucleotide variations), three per eggplant chromosome, were selected for validation in the S. incanum and S. aethiopicum accessions using the HRM (High Resolution Melting) technique [89]. Of these, a total of $96 \%$ were correctly amplified and in all of them polymorphisms detected have been confirmed (see Additional file 12).

For each of the four species, the intraspecific and interspecific polymorphisms were identified by filtering the VCF (Variant Call Format) file through the species ID number. In addition the INDELs were separated from the SNPs applying the VKS filter (it is not a SNP). The results of SNP calling are reported in Table 2. S. aethiopicum presented the highest value of intraspecific SNVs, with 159,571 SNPs and 4,556 INDELs. Many less intraspecific polymorphisms (12,396, of which 11,861 were SNPs and 535 INDELs) were identified in S. incanum. This suggests that the $S$. aethiopicum accession used presented a larger degree of heterozygosity than the S. incanum accession. Finally, in S. melongena and S. torvum [33] 2780 SNVs (2660 SNPs and 120 INDELs) and 25,147 SNVs (18,829 SNPs and 6,318 INDELs) intraspecific polymorphisms were discovered respectively. The high level of intraspecific variation in S. aethiopicum, $S$. torvum and S. incanum in comparison with S. melongena are probably due to the higher degree of autogamy and breeding selection for uniformity of the latter [90].

The interspecific SNVs were detected in the comparisons between two species or three species at the same time. An interspecific SNV were selected when the species
Table 2 Single nucleotide variations statistics for the S. incanum and S. aethiopicum transcriptomes

\begin{tabular}{|c|c|c|c|}
\hline SNVs intraspecific variations & INDELS & SNPS & Total SNVs \\
\hline S. incanum & 535 & 11,861 & 12,396 \\
\hline filtered & 28 & 385 & \\
\hline S. aethiopicum & 4,556 & 159,571 & 164,127 \\
\hline filtered & 312 & 5,804 & \\
\hline S. melongena & 120 & 2,660 & 2,780 \\
\hline filtered & 6 & 57 & \\
\hline S. torvum & 6,318 & 18,829 & 25,147 \\
\hline filtered & 26 & 90 & \\
\hline SNVs interspecific variations & INDELS & SNPS & Total SNVs \\
\hline S. incanum and S. aethiopicum & 586 & 14,576 & 15,162 \\
\hline filtered & 29 & 649 & \\
\hline S. incanum and S. melongena & 3,673 & 102,104 & 105,777 \\
\hline filtered & 253 & 4,184 & \\
\hline S. incanum and S. torvum & 96,799 & 491,965 & 588,764 \\
\hline filtered & 760 & 3,995 & \\
\hline S. aethiopicum and S. melongena & 2,392 & 73,059 & 75,451 \\
\hline filtered & 165 & 3,277 & \\
\hline S. aethiopicum and S. torvum & 83,106 & 420,685 & 503,791 \\
\hline filtered & 604 & 3,229 & \\
\hline S. melongena and S. torvum & 92,323 & 464,071 & 556,394 \\
\hline filtered & 722 & 3,682 & \\
\hline $\begin{array}{l}\text { S. incanum, S. aethiopicum } \\
\text { and S. melongena }\end{array}$ & 108 & 41 & 149 \\
\hline filtered & 8 & 2 & \\
\hline $\begin{array}{l}\text { S. incanum, S. aethiopicum } \\
\text { and S. torvum }\end{array}$ & 908 & 198 & 1,106 \\
\hline filtered & 10 & 3 & \\
\hline $\begin{array}{l}\text { S. incanum, S. melongena } \\
\text { and S. torvum }\end{array}$ & 8,022 & 1,265 & 9,287 \\
\hline filtered & 118 & 15 & \\
\hline $\begin{array}{l}\text { S. aethiopicum, S. melongena } \\
\text { and S. torvum }\end{array}$ & 6,986 & 886 & 7,872 \\
\hline filtered & 102 & 12 & \\
\hline $\begin{array}{l}\text { S. incanum, S. aethiopicum, } \\
\text { S. melongena and S. torvum }\end{array}$ & 50 & 0 & 50 \\
\hline filtered & 2 & 0 & \\
\hline
\end{tabular}

SNVs (SNPs and INDELs) have been identified by the Freebayes SNP caller. The interspecific and intraspecific variations, as well as all species combination, have been determined by filtering ID (identification) species number. Every species and species comparison present an unambiguous identification number. The INDELs have been separated from SNPs applying the VKF filter to the total SNVs. The filtered SNPs and INDELs have been obtained by adding the CS60, HV0.05, CL60 and CEF filters

were homozygous for a specific allele, but different allele from one species to another (Table 2). The Circos plot in Fig. 5 shows the six combinations of interspecific SNVs comparison and the location of the variations in the eggplant genome. As in the case of unigenes distribution 


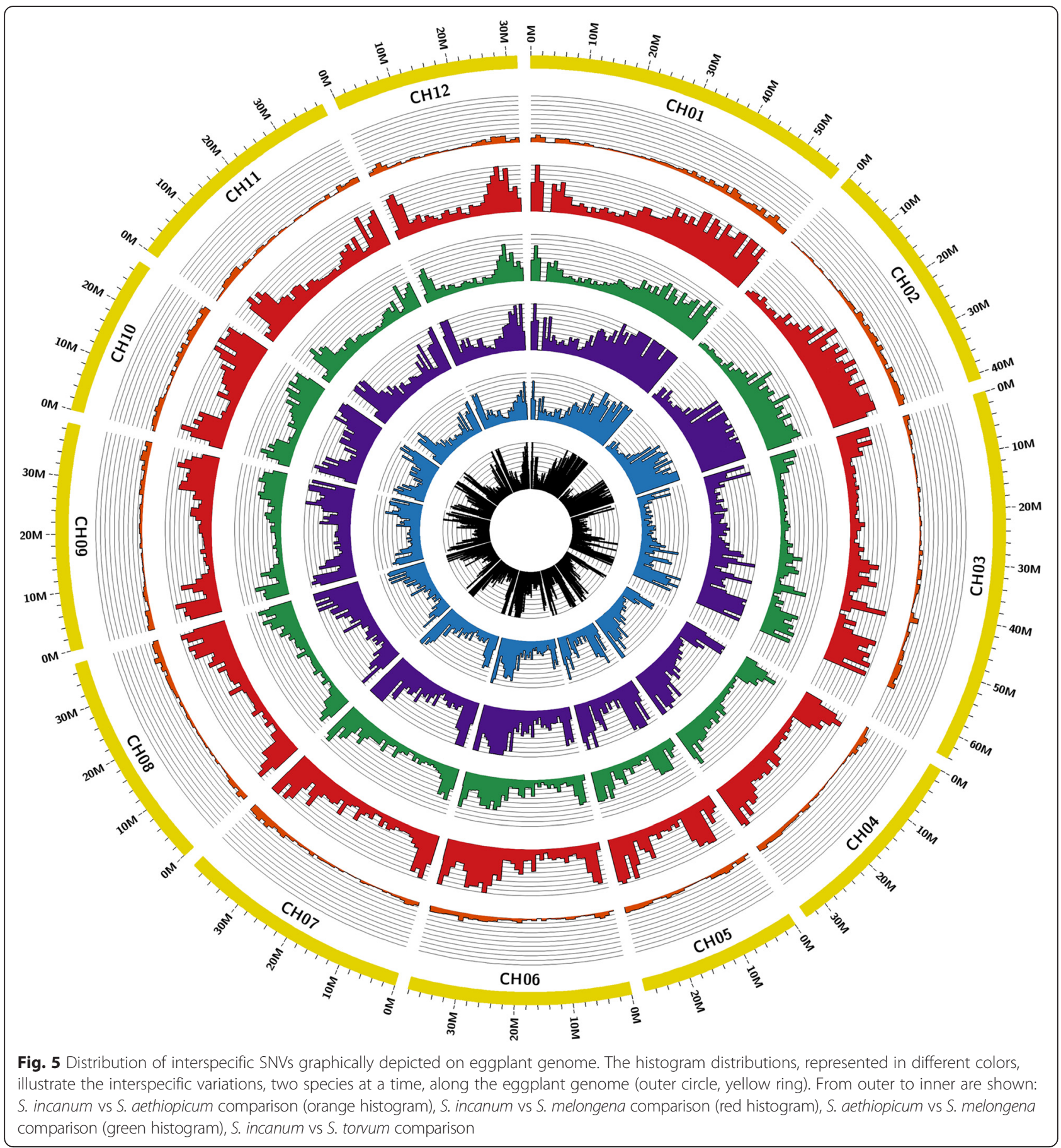

along the S. melongena genome, the SNVs are less represented in areas which could correspond to centromere and more represented in regions which could correspond to the chromosome arms. Solanum torvum presented the greater number of interspecific SNVs. A total of 1,648,949 polymorphisms (1,376,721 SNPs and 272,228 INDELs) have been detected in S. torvum, 588,764 of which are with S. incanum, 556,394 with S. melongena and 503,791 with $S$. aethiopicum. Out of the four species, $S$. torvum is the most phylogenetically distant [8]; this explains the large number of interspecific polymorphisms. Solanum melongena presented the second most abundant set of interspecific polymorphisms, 737,622 SNVs $(639,234$ SNPs and 98,388 INDELs). The comparison with $S$. aethiopicum detected 75,451 SNVs and 105,777 with S. incanum.

$S$. melongena and S. incanum, being members of the eggplant clade [8] we would expect less polymorphisms between these two species than between $S$. melongena 
and $S$. aethiopicum, which belongs to the anguivi clade. This discrepancy could be explained by the high amount of $S$. aethiopicum intraspecific polymorphisms, which reduces the differences in the number of SNVs between $S$. melongena and S. aethiopicum. Finally the lowest variation was found between $S$. incanum and S. aethiopicum (15,162 SNVs). The interspecific SNVs were substantially less abundant when three species were compared. The majority of polymorphisms were INDELs rather than SNPs, due to the preferential bi-allelic nature of the latter [91, 92]. The collections of variations have been larger in the combinations which included S. melongena and $S$. torvum, and smaller with $S$. incanum and $S$. aethiopicum. In addition 50 INDELs were detected when the four species were compared all together.

Subsequently, all intraspecific and interspecific SNVs detected in the four species were filtered in order to create subsets of the most suitable and effective variations for genotyping assays, both manually and with high throughput platforms, such as High Resolution Melting and GoldenGate Assay [89, 93, 94]. These variations have well-spaced positions, distanced more than $60 \mathrm{bp}$ from another polymorphism and from the edges of the assembled transcripts. Furthermore, they could be detected by digestion with commonly used and cheap restriction enzymes.

\section{SSRs}

The transcriptomes were examined to discover SSRs (Simple Sequence Repeat) made of di-, tri-, tetranucleotide motifs by using the Sputnik software [95]. In S. incanum, a set of 976 SSRs were identified in 954 unigenes; i.e., $1.1 \%$ of the unigenes contained at least one microsatellite, while in S. aethiopicum 1,708 SSRs were detected in 1628 unigenes (1.8\%). The total number of SSRs yielded was lower than obtained in other studies $[96,97]$, probably due to the stringent criteria used to obtain high confident markers. The microsatellites identified are summarized in Table 3, while their location on eggplant genome is shown in Fig. 2.

The range of SSRs length varied between 16 and 72 nucleotides in S. incanum and between 16 and 74 in $S$. aethiopicum with an average value of 21 and 24 nucleotides respectively. The most represented SSRs motifs in both species corresponded to AG, AAG and AAAG in agreement to the observation in other crops [98-100]. Trinucleotide repeat motifs were the most abundant (63.6\% and $62.7 \%$ ), followed by dinucleotide (26.6\% and $28.4 \%)$ and tetranucleotide $(9.8 \%$ and $8.9 \%)$ repeats. The prevalence of trinucleotide motifs is well documented in literature in eggplant $[29,101]$ as well as in other crops [102, 103]. Metzgar et al. [104] hypothesized that non-triplet SSRs show higher risks of frameshift mutation in coding regions and the selection against these mutations would reduce their chances of fixation.
Table 3 SSRs statistics corresponding to the S. incanum and S. aethiopicum transcriptomes

\begin{tabular}{|c|c|c|c|c|c|c|}
\hline \multirow[b]{2}{*}{ SSRs repeat motifs } & \multicolumn{3}{|c|}{ S. incanum } & \multicolumn{3}{|c|}{ S. aethiopicum } \\
\hline & SSRs & $\%$ & Unigenes & SSRs & $\%$ & Unigenes \\
\hline Dinucleotide & 260 & 26.6 & 258 & 362 & 29.0 & 342 \\
\hline $\mathrm{AG} / \mathrm{CT}$ & 160 & 61.5 & & 187 & 51.7 & \\
\hline AT/TA & 63 & 24.2 & & 98 & 27.1 & \\
\hline $\mathrm{AC} / \mathrm{GT}$ & 36 & 13.8 & & 76 & 20.9 & \\
\hline $\mathrm{CG} / \mathrm{GC}$ & 1 & 0.3 & & 1 & 0.3 & \\
\hline Trinucleotide & 621 & 63.6 & 609 & 776 & 62.2 & 755 \\
\hline $\mathrm{AAG} / \mathrm{CTT}$ & 169 & 27.2 & & 263 & 33.8 & \\
\hline AAC/GTT & 145 & 23.3 & & 173 & 22.2 & \\
\hline AAT/ATT & 82 & 13.2 & & 106 & 13.7 & \\
\hline ATC/GAT & 73 & 11.7 & & 63 & 8.1 & \\
\hline AGG/CCT & 53 & 8.5 & & 54 & 7.0 & \\
\hline ACC/GGT & 39 & 6.2 & & 41 & 5.3 & \\
\hline AGC/GCT & 34 & 5.4 & & 40 & 5.2 & \\
\hline $\mathrm{CCG} / \mathrm{CGG}$ & 12 & 1.9 & & 17 & 2.2 & \\
\hline $\mathrm{ACT} / \mathrm{AGT}$ & 10 & 1.6 & & 10 & 1.3 & \\
\hline ACG/CGT & 4 & 0.6 & & 9 & 1.2 & \\
\hline Tetranucleotide & 95 & 9.8 & 93 & 110 & 8.8 & 110 \\
\hline AAAG/CTTT & 29 & 30.5 & & 36 & 32.7 & \\
\hline AAAT/TTTA & 23 & 24.2 & & 28 & 25.5 & \\
\hline AAAC/GTT & 10 & 10.5 & & 5 & 4.5 & \\
\hline ACAT/ATGT & 5 & 5.2 & & 9 & 8.2 & \\
\hline AAGG/CCTT & 4 & 4.2 & & 3 & 2.8 & \\
\hline ATCC/GGAT & 4 & 4.2 & & 1 & 0.9 & \\
\hline AACC/GGTT & 3 & 3.1 & & 4 & 3.6 & \\
\hline AATG/CATT & 3 & 3.1 & & 2 & 1.8 & \\
\hline AATT/AATT & 3 & 3.1 & & 6 & 5.5 & \\
\hline AGGG/CCCT & 3 & 3.1 & & 1 & 0.9 & \\
\hline Others motifs & 8 & 8.4 & & 15 & 13.6 & \\
\hline Total & 976 & & 954 & 1,248 & & 1,270 \\
\hline
\end{tabular}

$\mathrm{Di}$-, tri- and tetranucleotide repeats and motifs identified in the S. incanum and $S$. aethiopicum assembled unigenes are indicated

Otherwise the selection against frameshift events does not occur in SSRs with a repeat length divisible by three (tri- and hexanucleotide repeats). Depending on their position in the gene, SSRs can be involved in different processes. The genic SSRs in $5^{\prime}$-UTR are implicated in gene transcription and gene translation while in $3^{\prime}$-UTR are implied in gene silencing and transcription slippage. In introns, SSRs can activate and inactivate genes [105]. The analysis of localization revealed that most of SSRs were located in ORFs, $33.5 \%$ for S. incanum and $32.7 \%$ for S. aethiopicum, and less in the UTRs (Table 4). In ORFs the trinucleotides repeats were the most abundant (88.7 \% for S. incanum and 87.7 for S. aethiopicum), 
Table 4 SSRs localization in the S. incanum and S. aethiopicum transcriptomes

\begin{tabular}{|c|c|c|c|c|c|c|c|c|}
\hline \multirow[t]{2}{*}{ S. incanum } & \multicolumn{2}{|c|}{ di-SSRs } & \multicolumn{2}{|c|}{ tri-SSRs } & \multicolumn{2}{|c|}{ tetra-SSRs } & \multicolumn{2}{|l|}{ Total } \\
\hline & $\mathrm{N}^{\circ}$ & $\%$ & $\mathrm{~N}^{\circ}$ & $\%$ & $\mathrm{~N}^{\circ}$ & $\%$ & $\mathrm{~N}^{\circ}$ & $\%$ \\
\hline $5^{\prime}-$ UTR & 63 & 42.2 & 66 & 44.3 & 20 & 13.5 & 149 & 15.2 \\
\hline ORF & 25 & 7.6 & 291 & 88.7 & 11 & 3.7 & 327 & 33.5 \\
\hline $3^{\prime}-U T R$ & 40 & 28.8 & 83 & 59.7 & 16 & 11.5 & 139 & 14.2 \\
\hline Other & 132 & 36.5 & 181 & 50.1 & 48 & 13.4 & 361 & 37.1 \\
\hline Total & 260 & 26.6 & 621 & 63.6 & 95 & 9.8 & 976 & 100 \\
\hline \multicolumn{2}{|c|}{ S. aethiopicum di-SSRs } & \multicolumn{2}{|c|}{ tri-SSRs } & \multicolumn{2}{|c|}{ tetra-SSRs } & \multicolumn{3}{|c|}{ Total } \\
\hline & $\mathrm{N}^{\circ}$ & $\%$ & $\mathrm{~N}^{\circ}$ & $\%$ & $\mathrm{~N}^{\circ}$ & $\%$ & $\mathrm{~N}^{\circ}$ & $\%$ \\
\hline $5^{\prime}-$ UTR & 74 & 43.0 & 75 & 43.6 & 23 & 13.4 & 172 & 13.8 \\
\hline ORF & 29 & 7.1 & 358 & 87.8 & 21 & 5.1 & 408 & 32.7 \\
\hline $3^{\prime}-$ UTR & 73 & 38.0 & 106 & 52.3 & 13 & 6.7 & 192 & 15.3 \\
\hline Other & 186 & 39.0 & 237 & 49.8 & 53 & 11.2 & 476 & 38.2 \\
\hline Total & 362 & 29.0 & 776 & 62.2 & 110 & 8.8 & 1,248 & 100 \\
\hline
\end{tabular}

The SSRs detected in the transcriptomes were checked for their position in the unigenes (ORF, $5^{\prime} \mathrm{UTR}$ and $\mathrm{3}^{\prime} \mathrm{UTR}$ ) according to the nucleotide repeats. When no precise position was established the SSRs were defined as "Other"

ensuring the conservation of coding capacity and better protection against big changes in frameshift which might cause dramatic effects. On the other hand dinucleotides and tetranucleotides were more abundant in the UTRs, showing no great differences between the $5^{\prime}$ and $3^{\prime}$. These results are consistent with previous study in other species $[102,106]$.

One polymorphic SSR was selected per chromosome to be validated in S. incanum and S. aethiopicum accessions used for the transcriptome sequencing. Eleven out of the 12 markers were correctly amplified and resulting polymorphic between the two species (Additional file 12). This indicates that the SSRs discovered in this study will provide a valuable set of molecular markers to disclose the intraspecific and interspecific variability across the eggplant genepool. The high rate of SNPs and SSRs correctly amplified is an indirect evaluation of good transcriptome assembly.

Genic SSRs present some advantages in comparison with genomic DNA-based markers. For instance, their development from RNA-Seq projects is low-cost [107]. Genic SSRs are in many cases functional markers, when changes in allelic repeats affect functions and the phenotype. Functional markers permit a direct allele selection, if there is a clear association for a target trait. Furthermore, the flanking sequences of genic SSRs are more evolutionary conserved than genomic SSRs [108, 109], allowing considerable cross-species transferability. On the basis of its properties, the uses of genic SSRs are multiple, allowing genome and comparative mapping, genetic diversity analysis, QTL studies, gene tagging, association mapping and functional genomics $[110,111]$.

\section{Conclusions}

In this study we present a de novo assembly and analysis of $S$. incanum and S. aethiopicum transcriptomes obtained by RNA-Seq. As a result of the annotation of these two common eggplant relatives, a broad overview of expressed genes was obtained. The annotation of the two transcriptomes has provided valuable information on function and structure of the assembled unigenes, which will allow the detection of candidate genes for important breeding traits in eggplant genepool. The large amount of intraspecific and interspecific molecular markers, genic SSRs and SNVs, identified in our transcriptomes and in the S. melongena and S. torvum transcriptomes [33], will be extremely helpful for the breeding programs, although a deeper comparison between the four transcriptome it would be of great interest. In particular filtered SNVs will allow accurate genotyping assays through high throughput platforms or arrays increasing the efficiency and rapidity of the programs. Overall, the information produced in this study provides a valuable genomic resource in two nonmodel species, opening the door to further studies as gene tagging, comparative mapping, association mapping for enhancing eggplant genomics and breeding.

\section{Methods}

\section{Plant material}

The materials used were S. incanum accession MM577, which was collected in the wild in Israel and S. aethiopicum accession BBS135, which belongs to the Gilo cultivar group and was originally collected in Ivory Coast. Solanum incanum accession MM577 is a spiny plant with small green rounded fruit and purple corolla while the $S$. aethiopicum accession BBS135 is thornless and presents green obovoid fruits and white corolla (Fig. 6). Solanum incanum accession MM577 has been used by our group as a parental to develop an interspecific genetic linkage map with Solanum melongena in which the candidate genes involved in the core chlorogenic acid synthesis pathway were mapped [20].

Plants of both accessions were grown in a greenhouse at the Universitat Politècnica de València (Valencia, Spain). Tissue samples were taken all at once at the stages of young leaf, floral bud and young fruit from a single plant and immediately frozen in liquid nitrogen and stored at$80{ }^{\circ} \mathrm{C}$ until used for RNA extraction.

\section{RNA extraction for Illumina sequencing}

About $100 \mathrm{mg}$ of tissue were powdered in liquid nitrogen with a mortar and pestle. TRI Reagent ${ }^{\circ}$ Protocol (SigmaAldrich, St. Louis, USA) was used for the total RNA extraction. In order to avoid DNA contamination DNase I Recombinant, RNase-free (Roche, Basel, Switzerland) was used. RNA integrity was confirmed by agarose electrophoresis and RNA quantification was performed using a 

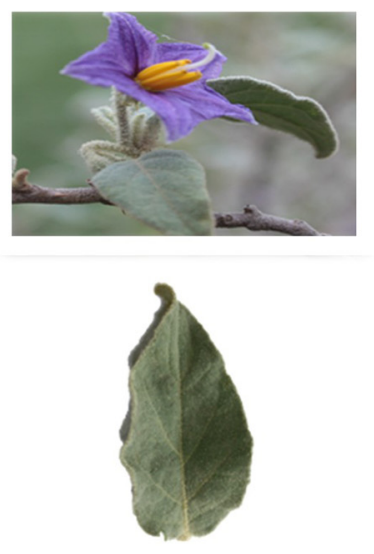

Solanum incanum MM577
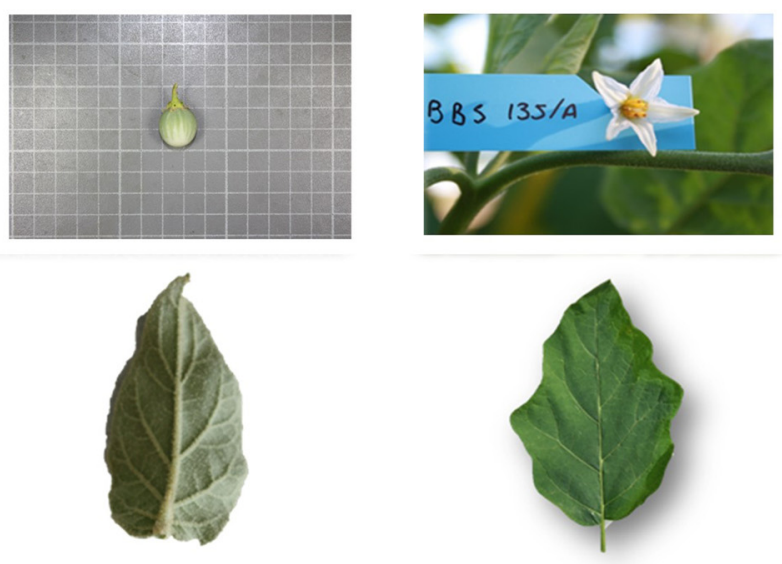

Solanum aethiopicum BBS135

Fig. 6 Flower, fruit and leaf of S. incanum (left) and S. aethiopicum (right). The cells in the fruit picture grid have a size of $1 \mathrm{~cm} \times 1 \mathrm{~cm}$

Nanodrop ND-1000 spectrophotometer (Thermo Scientific, Wilmington, USA). Equal amounts of total RNA from each tissue were pooled for each accession and send to Macrogen Korea (Seoul, South Korea). After the construction of paired-end library (insert size of $300 \mathrm{bp})$, RNA-Seq was performed in HiSeq 2000 sequencer (Illumina, San Diego, USA). The raw sequences obtained are available in the Sequence Read Archive at NCBI [35] at the accession number (SRS1054263) for $S$. incanum and at the accession number (SRS1052489) for S. aethiopicum.

\section{Sequence data analysis and De novo assembly}

The quality of the reads generated by Illumina was checked using the FastQC program [112]. In order to obtain high-quality data, the raw reads were pre-processed and trimmed using in-house developed software, NGS CRUMBS [113]. Through the different utilities the adapters used during the sequencing process were removed, as well as, low quality sequences, with a Phred quality score $\mathrm{Q}<20$ and ambiguous sequences with $\mathrm{N}$. The trimmed reads were finally assembled into transcripts using Trinity [50], using default setting, which was specifically developed for de novo transcriptome assembly and for short-read RNA-Seq like Illumina HiSeq 2000.

In order to reduce the redundancy, the assembled transcriptomes were screened with CAP3 program [114]. CAP3 uses base quality values, merging transcripts which overlap at least $200 \mathrm{bp}$ with an identity of $99 \%$. After that, to remove low complex sequences, the transcripts which have shown a DUST score less than 7 were masked [115]. The estimation of transcript expression levels were calculated using RSEM software [55] and subsequently the most expressed transcripts of each Trinity transcript cluster were selected to create a set of unigenes for each species. Then both sets of unigenes were blasted (cut-off value of 1e-20) against the eggplant genome in order to obtain the physical position. The distribution of unigenes over eggplant genome was graphically depicted with Circos software [56].

\section{Structural and functional annotation}

The set of assembled transcripts was compared using BlastX (cut-off value of 1e-20) against four public protein databases in the following order: Swiss-Prot [64], ITAG2.4 [65], Arabidopsis [66] and UniRef90 [67]. If a transcript gave a blast hit in the first database, no further searches were done, otherwise a second, third or fourth blast was performed.

Subsequently, a functional annotation was realized using Blast2GO software [68] to assign at the transcripts the corresponding GO terms [116] and EC number [78]. For this purpose a BlastX (cut-off value of 1e-20) was performed in the NR database [35] and the resulting hits were mapped into gene ontology database to assign the correspondent annotation. Blast2GO was used also to obtain the KEGGs pathways from the Kyoto Encyclopedia of Genes and Genomes database (version 73.0, January 1, 2015) [80].

Additionally, best reciprocal hits with BlastN (cut-off value of 1e-20) were performed with tomato genome (version SL2.50) [69] to detect orthologs. On the other hand the tomato genome was employed to predict gene model and intron frames, using est2genome software [82]. ORFs were predicted with ESTScan program [81].

\section{Mapping transcriptomes against eggplant genome}

The high-quality clean reads from our RNA-Seq experiment (S. incanum and S. aethiopicum) were aligned against the eggplant genome using the Top Hat program [117]. The TopHat pipeline is very fast and specifically designed for detecting junctions even in genes transcribed 
at very low levels. Because only $20 \%$ of S. torvum reads mapped in eggplant genome with Top Hat, we decide to use a BWA [51], a most suited mapper in the case of greater genetic distance. Subsequently, the reads were realigned using the GATK (Genome Analysis Tool Kit) software in order to split the reads [118].

Raw paired-end reads from Solanum melongena and $S$. torvum were downloaded through the NCBI Sequence Read Archive (SRA). The S. melongena reads were deposited under accession number [SRA: SRR1104129] and S. torvum reads under accession [SRA: SRR1104128]. The raw sequences were processed and trimmed as described above. The FASTA sequence of the draft eggplant genome was downloaded from the Eggplant Genome Database [119].

\section{Molecular markers discovery \\ SNVS}

SNVs (SNPs and INDELs) were detected using the FreeBayes program [88], a bayesian haplotype-based SNP caller, using the Top Hat alignment. To verify the quality of SNP calling, three SNVs per eggplant chromosome were validated in the sequenced genotypes. Every SNV locus was checked by IGV software [120], to select the most polymorphic loci with the higher coverage. Primers pairs were designed in flanking regions using Primers3 [121].

HRM-based PCR was used to validate the SNPs in a LightCycler 480 Real-Time PCR (Roche, Basel, Switzerland). The reactions were performed in a $10 \mu \mathrm{L}: 5 \mu \mathrm{L}$ Master Mix $2 \times, 0.8 \mu \mathrm{L} \mathrm{MgCl} 225 \mathrm{mM}, 0.25 \mu \mathrm{L}$ each primer, $1.7 \mu \mathrm{L}$ water and $2 \mu \mathrm{L}$ DNA $30 \mathrm{ng} / \mu \mathrm{L}$ with the following touchdown PCR program: denaturation at $95{ }^{\circ} \mathrm{C}$ for $10 \mathrm{~min}$, followed by 55 cycles of $10 \mathrm{~s}$ at $95{ }^{\circ} \mathrm{C}, 15 \mathrm{~s}$ at $65{ }^{\circ} \mathrm{C}$ (decreasing $1{ }^{\circ} \mathrm{C}$ each cycle until $55^{\circ} \mathrm{C}$ ) and of $15 \mathrm{~s}$ at $72{ }^{\circ} \mathrm{C}$, finally the melting at $1 \mathrm{~min}$ at $95{ }^{\circ} \mathrm{C}$, $1 \mathrm{~min}$ at $40{ }^{\circ} \mathrm{C}, 1 \mathrm{sec}$ at $60{ }^{\circ} \mathrm{C}$ and rising the temperature at $0.02{ }^{\circ} \mathrm{C} / \mathrm{s}$ until $95^{\circ} \mathrm{C}$.

Although all SNVs matched the quality criteria, not all of them seemed equally reliable. Different filters, developed by ours [113], have been applied to VCF file in order to maximize the polymorphism validation. The settings of the filters are provided in the Additional file 13. The VKS filter was applied to differentiate INDELs from SNPs, the filter CS60 to detect if the SNV was closer than 60 nucleotides to another SNV, the filter CL60 to identify SNVs closer than 60 nucleotides to the transcript edge and the filter HV0.05 to determine if the region had more than 5 SNVs per 100 bases. All these filters allow selecting SVNs, with small amplicons size (e.g. 80-100 bp) and are suitable for manually validation such as with HRM as well as for high-throughput genotyping platform [89, 93, 94]. If the SNV is going to be genotyped by CAPS, the filter CEF will help to select common low-priced digestion enzymes.
SSRs

The annotation of SSRs was carried out with Sputnik software [95], selecting the sequences containing $\geq 9$ di-, $\geq 6$ tri-, or $\geq 4$ tetranucleotide motifs. The sequences of unigenes which contain SSRs were blasted against the eggplant genome database in order to know their physical position while their region in the transcripts (ORFs, 3 '-UTR and 5' -UTR) were detected using the Bedtools utilities [122]. The representation of the SSRs distribution along the eggplant genome was performed with Circos software. One SSR per eggplant chromosome was selected in order to validate them in the S. incanum and $S$. aethiopicum sequenced genotypes.

All selected SSRs for validation were checked via IGV viewer and primers pairs were designed with Primers3. The amplification of SSRs were performed by touchdown PCR in a final volume of $12 \mu \mathrm{L}: 7.21 \mu \mathrm{L}$ water, $1.2 \mu \mathrm{L} 1 \times$ PCR buffer, $0.6 \mu \mathrm{L} \mathrm{MgCl}_{2} 50 \mathrm{mM}, 0.24 \mu \mathrm{L}$ dNTPs $10 \mathrm{mM}, 0.3 \mu \mathrm{L}$ reverse primer $10 \mu \mathrm{M}, 0.06 \mu \mathrm{L}$ forward primer with M13 tail $10 \mu \mathrm{M}, 0.24 \mu \mathrm{L}$ fluorochrome (FAM, VIC, NED and PET) $10 \mu \mathrm{M}, 0.15 \mu \mathrm{L}$ Taq DNA Polymerase $(5 \mathrm{U} / \mu \mathrm{L}), 2 \mu \mathrm{L}$ DNA template $20 \mathrm{ng} / \mu \mathrm{L}$ under the following cycling conditions: denaturation at $95{ }^{\circ} \mathrm{C}$ for $3 \mathrm{~min}$, followed by 10 cycles of $30 \mathrm{~s}$ at $95{ }^{\circ} \mathrm{C}$, $30 \mathrm{~s}$ at $65^{\circ} \mathrm{C}$ (with each cycle the annealing temperature decreasing $1{ }^{\circ} \mathrm{C}$ ), and of $30 \mathrm{~s}$ at $72{ }^{\circ} \mathrm{C}$. Products were subsequently amplified for 20 cycles at $95{ }^{\circ} \mathrm{C}$ for $30 \mathrm{~s}$, $55{ }^{\circ} \mathrm{C}$ for $30 \mathrm{~s}$ and $72{ }^{\circ} \mathrm{C}$ for $30 \mathrm{~s}$, with a final extension at $72{ }^{\circ} \mathrm{C}$ for $5 \mathrm{~min}$.

PCR products were diluted in formamide and analyzed on an automated DNA sequencer ABI PRISM 3100Avant with a GeneScan 600LIZ (Applied Biosystems, California, USA) size standard. The data were analyzed using the GeneScan software (Applied Biosystems) to obtain the electropherograms and polymorphisms were analyzed with Genotyper DNA Fragment Analysis software (Applied Biosystems, California, USA).

\section{Ethics and consent to participate}

Not Applicable.

\section{Consent to publish \\ Not Applicable.}

\section{Availability of data and materials}

The datasets supporting the conclusions of this article are available in the Sequence Read Archive at the National Center for Biotechnology Information (NCBI) at the accession number SRS1054263 (http://www.ncbi. nlm.nih.gov/sra?LinkName=biosample_sra\&from_uid $=402$ 3348 ) for $S$. incanum and at the accession number SRS1052489 (http://www.ncbi.nlm.nih.gov/sra?LinkName= biosample_sra\&from_uid=4025429) for S. aethiopicum. 
The datasets supporting the conclusions of this article are included within the article and its additional files.

\section{Additional files}

Additional file 1: Solanum incanum and S. aethiopicum assembled transcripts (compressed folder). The file provides the fasta sequences of the 108,322 S. incanum and 106,660 S. aethiopicum transcripts. (7Z $38772 \mathrm{~kb})$

Additional file 2: List of Solanum incanum and S. aethiopicum unigenes (compressed folder). The file provides the list of the 83,905 most expressed single-copy S. incanum and 87,084 S. aethiopicum transcripts in fasta format. (7Z $29593 \mathrm{~kb}$ )

Additional file 3: Solanum incanum and S. aethiopicum unigene physical positions (compressed folder). Blast search results in order to obtain the S. incanum and S. aethiopicum unigen positions using S. melongena draft genome (cut-off e-value 1e-20). (7Z 22726 kb)

Additional file 4: S. incanum and S. aethiopicum unigenes potentially encoding proteins (compressed folder). The file contains the unigene protein annotations performed using the three major public and the tomato protein databases (Swiss-Prot, ITAG 2.4, Arabidopsis, Uniref90). (7Z $1181 \mathrm{~kb})$

Additional file 5: $\mathrm{GO}$ terms and EC number unigene annotation (compressed folder). The file provides a list of GO terms and EC numbers assigned to S. incanum and S. aethiopicum unigenes. (7Z $1086 \mathrm{~kb}$ )

Additional file 6: Biological pathway maps (compressed folder). The file provides the biological pathway maps, obtained by KEGG searches. (7Z $8001 \mathrm{~kb}$ )

Additional file 7: Details of KEGG annotation (compressed folder). The files provide the names of biological pathways, number of unigenes involved, enzymes types and EC numbers of S. incanum and S. aethiopicum unigenes KEGG annotation. (7Z 56 kb)

Additional file 8: Position in tomato reference genome (compressed folder). The file provides the physical position of S. incanum and S. aethiopicum unigenes in tomato through Blast against S. Iycopersicum genome, version SL2.5. (7Z $450 \mathrm{~kb}$ )

Additional file 9: Solanum incanum and S. aethiopicum orthologs (compressed folder). The file provides the lists of S. incanum and S. aethiopicum unigenes which presented orthologs in S. lycopersicum obtained through best reciprocal hits using BlastN. (7Z 524 kb)

Additional file 10: ORFs and introns annotation (compressed folder). The GFF3 format file provides the ORFs and introns detected in $S$. incanum and S. aethiopicum unigenes. (7Z $6710 \mathrm{~kb}$ )

Additional file 11: SNP calling results. The file in VCF format provides the list of SNVs (SNPS and INDELS) identified in S. incanum, S. aethiopicum, S. melongena and S. torvum transcriptomes by mapping against S. melongena genome. For each SNV are indicated their position in eggplant genome scaffold, the allele of reference (eggplant genome allele) and the alternative allele (the transcriptomes allele), the quality of the SNV, the filters applied and detailed information about SNP calling process for each SNV and the different kind of transcriptome ID combinations in order to filter the intraspecific and interspecific polymorphisms. The different filter applied for in silico selection were: CS60 (the SNV is closer than 60 nucleotides to another SNV), HV0.05 (the region has more than 5.0 SVNs per 100 bases), CL60 (the SNV is closer than 60 nucleotides to the reference edge), CEF (SNV is not a CAP detectable by the enzyme: cheap_ones), VKF (it is not an SNP). (7Z 38609 kb)

Additional file 12: Validated markers. The file provides the tables of SNPS and SSRs experimentally validated. For each marker are provided the unigene which came from, the position in the correspondent eggplant scaffold, the detected alleles and the primers used for validation. (XLSX $21 \mathrm{~kb}$ )

Additional file 13: Filters settings. The file provides the settings of the filters that have been applied to VCF file in order to filter the SNVs. (TXT $1 \mathrm{~kb}$ )

\section{Abbreviations}

EC number: enzyme commission number; ESTs: Expressed sequence tag: FISH: fluorescence in situ hybridization; GATK: genome analysis tool kit; GO term: gene ontology term; HRM: high resolution melting; INDEL: insertion/ deletions; KEGG: kyoto encyclopedia of genes and genomes; NCBI: national center for biotechnology information; ncRNAs: noncoding RNAs; NGS: nextgeneration sequencing; NR database: non-redundant protein database; ORF: open reading frame; SNP: single nucleotide polymorphism; SNVs: single nucleotide variations; SSR: simple sequence repeat; UTR: untranslated region; VCF: variant call format.

\section{Competing interests}

The authors declare that they have no competing interests.

\section{Authors' contributions}

PG, JB, PZ and SV performed the bioinformatic analyses. JP and SV conceived the study. FJH and MP contributed to the data analysis. PG and MP participated in plant material preparations. FJH and JB contributed to the discussion. PG, SV and JP drafted the manuscript. All authors read and approved the final manuscript.

\section{Acknowledgements}

The authors thankfully acknowledge the computer resources, technical expertise and assistance provided by the PAB (Andalusian Bioinformatics Platform) center located at the SCBI of the University of Malaga (http:// www.scbi.uma.es/site/). Pietro Gramazio is grateful to the Universitat Politècnica de València for a pre-doctoral (Programa FPI de la UPVSubprograma 1/2013 call) contract.

\section{Funding}

This work has been partially funded by Spanish Ministerio de Economía y Competitividad and FEDER (grant AGL2015-64755-450 R).

Received: 22 September 2015 Accepted: 19 April 2016

Published online: 23 April 2016

References

1. The FAOSTAT Database. [http://faostat.fao.org/]. Accessed 20 September 2015.

2. Collonnier C, Fock I, Kashyap V, Rotino G, Daunay M, Lian Y, et al. Applications of biotechnology in eggplant. Plant Cell Tiss Org. 2001;65(2):91-107.

3. Plazas M, Andújar I, Vilanova S, Gramazio P, Herraiz FJ, Prohens J. Conventional and phenomics characterization provides insight into the diversity and relationships of hypervariable scarlet (Solanum aethiopicum L.) and gboma (S. macrocarpon L.) eggplant complexes. Front Plant Sci. 2014. doi:10.3389/fpls.2014.00318.

4. Rotino GL, Sala T, Toppino L. Eggplant. In: Pratap A, Kumar J, editors. Alien Gene Transfer in Crop Plants, vol. 2. New York: Springer; 2014. p. 381-409.

5. Meyer RS, Karol KG, Little DP, Nee MH, Litt A. Phylogeographic relationships among Asian eggplants and new perspectives on eggplant domestication. Mol Phylogenet Evol. 2012;63(3):685-701.

6. Schippers RR. African indigenous vegetables: an overview of the cultivated species. Wallingford: UK: CAB International; 2000.

7. Maundu P, Achigan-Dako E, Morimoto Y. Biodiversity of African vegetables. In: Shackleton CM, Pasquini MW, Drescher AW, editors. African indigenous vegetables in urban agriculture. UK: MapSet Ltd; 2009. p. 65-104.

8. Vorontsova M, Stern S, Bohs L, Knapp S. African spiny Solanum (subgenus Leptostemonum, Solanaceae): a thorny phylogenetic tangle. Bot J Linn Soc. 2013;173(2):176-93.

9. Rizza F, Mennella G, Collonnier C, Sihachakr D, Kashyap V, Rajam M, et al. Androgenic dihaploids from somatic hybrids between Solanum melongena and S. aethiopicum group gilo as a source of resistance to Fusarium oxysporum f. sp. melongenae. Plant Cell Rep. 2002;20(11):1022-32.

10. Mennella G, Rotino GL, Fibiani M, D'Alessandro A, Francese G, Toppino L, et al. Characterization of health-related compounds in eggplant (Solanum melongena L.) lines derived from introgression of allied species. J Agric Food Chem. 2010;58(13):7597-603.

11. Prohens J, Plazas M, Raigón MD, Seguí-Simarro JM, Stommel JR, Vilanova S. Characterization of interspecific hybrids and first backcross generations from crosses between two cultivated eggplants (Solanum melongena and S. 
aethiopicum Kumba group) and implications for eggplant breeding. Euphytica. 2012;186(2):517-38.

12. Prohens J, Whitaker BD, Plazas M, Vilanova S, Hurtado M, Blasco M, et al. Genetic diversity in morphological characters and phenolic acids content resulting from an interspecific cross between eggplant, Solanum melongena, and its wild ancestor (S. incanum). Ann Appl Biol. 2013;162(2):242-57.

13. Plazas M, Prohens J, Cuñat AN, Vilanova S, Gramazio P, Herraiz FJ, Andújar I. Reducing Capacity, Chlorogenic Acid Content and Biological Activity in a Collection of Scarlet (Solanum aethiopicum) and Gboma (S. macrocarpon) Eggplants. Int J Mol Sci. 2014;15(10):17221-41.

14. Lester RN, Hasan SMZ. Origin and domestication of the brinjal eggplant, Solanum melongena, from S. incanum, in Africa and Asia. In: Hawkes JG, Lester RN, Nee MH, Estrada N, editors. Solanaceae III: taxonomy, chemistry, evolution. London, U.K: Royal Botanic Gardens, Kew; 1991. p. 369-88.

15. Knapp S, Vorontsova MS, Prohens J. Wild relatives of the eggplant (Solanum melongena L.: Solanaceae): new understanding of species names in a complex group. PLoS One. 2013;8(2):e57039.

16. Stommel JR, Whitaker BD. Phenolic acid content and composition of eggplant fruit in a germplasm core subset. J Am Soc Hort Sci. 2003;128(5):704-10.

17. Yamakawa K, Mochizuki H. Nature and inheritance of Fusarium-wilt resistance in eggplant cultivars and related wild Solanum species. Bulletin of the Vegetable and Ornamental Crops Research Station. 1979;6:19-27.

18. Anis M, Baksh S, Iqbal M. Cytogenetic Studies on the F1 Hybrid Solanum incanum * S. melongena var. American Wonder. Cytologia. 1994;59(4):433-6.

19. Behera T, Singh N. Inter-specific crosses between eggplant (Solanum melongena L.) with related Solanum species. Sci. Hortic. 2002;95(1):165-72.

20. Gramazio P, Prohens J, Plazas M, Andujar I, Herraiz FJ, Castillo E, et al. Location of chlorogenic acid biosynthesis pathway and polyphenol oxidase genes in a new interspecific anchored linkage map of eggplant. BMC Plant Biol. 2014;14:350.

21. Schippers RR. African indigenous vegetables: an overview of the cultivated species. 2000

22. Sunseri F, Polignano GB, Alba V, Lotti C, Bisignano V, Mennella G, et al. Genetic diversity and characterization of African eggplant germplasm collection. Afr J Plant Sci. 2010;4(7):231-41.

23. Lester R, Niakan L. Origin and domestication of the scarlet eggplant, Solanum aethiopicum, from S. anguivi in Africa. In: D'Arcy WG, editor. Solanaceae: Biology and systematics. Columbia: Columbia University Press; 1986. p. 433-56.

24. Cappelli C, Stravato VM, Rotino GL, Buonaurio R. Sources of resistance among Solanum spp. to an Italian isolate of Fusarium oxysporum $\mathrm{fsp}$. Melongenae. Proceeding of the $9^{\text {th }}$ EUCARPIA meeting on genetics and breeding of capsicum and eggplant. 1995. p. 221-4.

25. Collonnier C, Mulya K, Fock I, Mariska I, Servaes A, Vedel F, et al. Source of resistance against Ralstonia solanacearum in fertile somatic hybrids of eggplant (Solanum melongena L.) with Solanum aethiopicum L. Plant Sci. 2001;160(2):301-13.

26. Gisbert C, Prohens J, Raigón MD, Stommel JR, Nuez F. Eggplant relatives as sources of variation for developing new rootstocks: Effects of grafting on eggplant yield and fruit apparent quality and composition. Sci Hortic. 2011;128(1):14-22.

27. Nunome T, Ishiguro K, Yoshida T, Hirai M. Mapping of Fruit Shape and Color Development Traits in Eggplant (Solanum melongena L.) Based on RAPD and AFLP Markers. Breed Sci. 2001;51(1):19-26.

28. Doganlar S, Frary A, Daunay MC, Lester RN, Tanksley SD. A comparative genetic linkage map of eggplant (Solanum melongena) and its implications for genome evolution in the Solanaceae. Genetics. 2002;161(4):1697-711.

29. Barchi L, Lanteri S, Portis E, Acquadro A, Vale G, Toppino L, et al. Identification of SNP and SSR markers in eggplant using RAD tag sequencing. BMC Genomics. 2011;12:304.

30. Fukuoka H, Miyatake K, Nunome T, Negoro S, Shirasawa K, Isobe S, et al. Development of gene-based markers and construction of an integrated linkage map in eggplant by using Solanum orthologous (SOL) gene sets. Theor Appl Genet. 2012;125(1):47-56.

31. Nunome T, Negoro S, Kono I, Kanamori H, Miyatake K, Yamaguchi H, et al. Development of SSR markers derived from SSR-enriched genomic library of eggplant (Solanum melongena L.). Theor Appl Genet. 2009;119(6):1143-53.

32. Vilanova S, Manzur JP, Prohens J. Development and characterization of genomic simple sequence repeat markers in eggplant and their application to the study of diversity and relationships in a collection of different cultivar types and origins. Mol Breed. 2012;30(2):647-60.
33. Yang X, Cheng YF, Deng C, Ma Y, Wang ZW, Chen XH, et al. Comparative transcriptome analysis of eggplant (Solanum melongena $\mathrm{L}$.) and turkey berry (Solanum torvum Sw.): phylogenomics and disease resistance analysis. BMC Genomics. 2014;15(1):412.

34. Hirakawa H, Shirasawa K, Miyatake K, Nunome T, Negoro S, Ohyama A, et al. Draft genome sequence of eggplant (Solanum melongena L.): the representative solanum species indigenous to the old world. DNA Res. 2014;21(6):649-60

35. The NCBI (National Center for Biotechnology Information) database. [http://www.ncbi.nlm.nih.gov/]. Accessed 20 September 2015.

36. Jaiswal B. Solanum torvum: a review of its traditional uses, phytochemistry and pharmacology. Int J Pharm Biol Sci. 2012;3:4.

37. Mohan M, Kamble S, Gadhi P, Kasture S. Protective effect of Solanum torvum on doxorubicin-induced nephrotoxicity in rats. Food Chem Toxicol. 2010:48(1):436-40.

38. Gandhi GR, Ignacimuthu S, Paulraj MG, Sasikumar P. Antihyperglycemic activity and antidiabetic effect of methyl caffeate isolated from Solanum torvum Swartz. fruit in streptozotocin induced diabetic rats. Eur J Pharmacol. 2011;670(2):623-31.

39. Gousset C, Collonnier C, Mulya K, Mariska I, Rotino GL, Besse P, et al. Solanum torvum, as a useful source of resistance against bacterial and fungal diseases for improvement of eggplant (S. melongena L.). Plant Sci. 2005;168(2):319-27.

40. Bagnaresi P, Sala T, Irdani T, Scotto C, Lamontanara A, Beretta M, et al. Solanum torvum responses to the root-knot nematode Meloidogyne incognita. BMC Genomics. 2013;14:540.

41. Guri A, Sink K. Interspecific somatic hybrid plants between eggplant (Solanum melongena) and Solanum torvum. Theor Appl Genet. 1988;76(4):490-6.

42. Sihachakr D, Haicour R, Chaput M, Barrientos E, Ducreux G, Rossignol L. Somatic hybrid plants produced by electrofusion between Solanum melongena L. and Solanum torvum Sw. Theor Appl Genet. 1989;77(1):1-6.

43. Collonnier C, Fock I, Mariska I, Servaes A, Vedel F, Siljak-Yakovlev S, et al. GISH confirmation of somatic hybrids between Solanum melongena and $S$. torvum: assessment of resistance to both fungal and bacterial wilts. Plant Physiol Biochem. 2003;41(5):459-70.

44. Kumchai J, Wei Y, Lee C, Chen F, Chin S. Production of interspecific hybrids between commercial cultivars of the eggplant (Solanum melongena $\mathrm{L}$.) and its wild relative S. torvum. Gen Mol Res. 2013;12(1):755-64.

45. YiKui W, YanYan F, Hong W, WenJia L, Yan L. Pollen development and anther callus induction in hybrid F1 by Solanum torvum SW. $(\hat{0}) \times$ Solanum melongena L. (P). Journal of Southern Agriculture. 2014;45(12):1967-71.

46. Takeda H, Sato A, Nishihara E, Arao T. Reduction of cadmium concentration in eggplant (Solanum melongena) fruits by grafting with Solanum torvum rootstock. Japanese Journal of Soil Science and Plant Nutrition. 2007.

47. Sabatino L, Palazzolo E, D'Anna F. Grafting suitability of Sicilian eggplant ecotypes onto Solanum torvum: Fruit composition, production and phenology. Int j food, agric and environ. 2013;11(3):1195-200.

48. Miceli A, Sabatino L, Moncada A, Vetrano F, D'Anna F. Nursery and field evaluation of eggplant grafted onto unrooted cuttings of Solanum torvum Sw. Sci Hort. 2014;178:203-10.

49. Davey JW, Blaxter ML. RADSeq: next-generation population genetics. Brief Funct Genomics. 2010;9(5-6):416-23.

50. Grabherr MG, Haas BJ, Yassour M, Levin JZ, Thompson DA, Amit I, et al. Fulllength transcriptome assembly from RNA-Seq data without a reference genome. Nat Biotechnol. 2011;29(7):644-52.

51. Li H. Aligning sequence reads, clone sequences and assembly contigs with BWA-MEM. arXiv preprint. arXiv. 2013;1303:3997.

52. Van Dijk EL, Auger $H$, Jaszczyszyn $Y$, Thermes $C$. Ten years of nextgeneration sequencing technology. Trends Genet. 2014;30(9):418-26.

53. Lee $\mathrm{S}$, Abecasis GR, Boehnke $\mathrm{M}$, Lin X. Rare-variant association analysis: study designs and statistical tests. Am J Hum Genet. 2014;95(1):5-23.

54. Faure D, Joly D. Next-generation sequencing as a powerful motor for advances in the biological and environmental sciences. Genetica. 2015;143(2):129-32.

55. Li B, Dewey CN. RSEM: accurate transcript quantification from RNA-Seq data with or without a reference genome. BMC Bioinformatics. 2011;12(1):323.

56. Krzywinski M, Schein J, Birol I, Connors J, Gascoyne R, Horsman D, et al. Circos: an information aesthetic for comparative genomics. Genome Res. 2009:19(9):1639-45.

57. Erayman M, Sandhu D, Sidhu D, Dilbirligi M, Baenziger PS, Gill KS. Demarcating the gene-rich regions of the wheat genome. Nucleic Acids Res. 2004;32(12):3546-65. 
58. Mizuno H, Kawahara Y, Wu J, Katayose $Y$, Kanamori H, Ikawa H, et al. Asymmetric distribution of gene expression in the centromeric region of rice chromosome 5. Front Plant Sci. 2011;2:16.

59. Fukuoka H, Yamaguchi H, Nunome T, Negoro S, Miyatake K, Ohyama A. Accumulation, functional annotation, and comparative analysis of expressed sequence tags in eggplant (Solanum melongena L.), the third pole of the genus Solanum species after tomato and potato. Gene. 2010;450(1):76-84.

60. Groves RA, Hagel JM, Zhang Y, Kilpatrick K, Levy A, Marsolais F, et al. Transcriptome profiling of khat (Catha edulis) and Ephedra sinica reveals gene candidates potentially involved in amphetamine-type alkaloid biosynthesis. PLoS One. 2015;10(3):e0119701.

61. Zhang J, Liu W, Han H, Song L, Bai L, Gao Z, et al. De novo transcriptome sequencing of Agropyron cristatum to identify available gene resources for the enhancement of wheat. Genomics. 2015;106(2):129-36.

62. Zhang S, Shi Y, Cheng N, Du H, Fan W, Wang C. De novo characterization of fall dormant and nondormant alfalfa (Medicago sativa L.) leaf transcriptome and identification of candidate genes related to fall dormancy. PLoS One. 2015;10(3):e0122170.

63. Mutz K, Heilkenbrinker A, Lönne M, Walter J, Stahl F. Transcriptome analysis using next-generation sequencing. Curr Opin Biotechnol. 2013;24(1):22-30.

64. The Swiss-Prot database. [http://web.expasy.org/docs/swiss-prot_guideline.html]. Accessed 20 September 2015.

65. The ITAG2.4 database. [ftp://ftp.sgn.cornell.edu/tomato_genome/annotation/]. Accessed 20 September 2015.

66. The TAIR database: The Arabidopsis Information Resource. [http://www. arabidopsis.org/]. Accessed 20 September 2015.

67. The UniRef90 database. [http://www.ebi.ac.uk/uniprot/database/download.html]. Accessed 20 September 2015.

68. Conesa A, Gotz S. Blast2GO: A comprehensive suite for functional analysis in plant genomics. Int J Plant Genomics. 2008;2008:619832.

69. Tomato Genome Consortium. The tomato genome sequence provides insights into fleshy fruit evolution. Nature. 2012;485(7400):635-41.

70. Ashrafi H, Hill T, Stoffel K, Kozik A, Yao J, Chin-Wo SR, et al. De novo assembly of the pepper transcriptome (Capsicum annuum): a benchmark for in silico discovery of SNPS, SSRs and candidate genes. BMC Genomics. 2012;13:571.

71. Bao Y, Xu S, Jing X, Meng L, Qin Z. De Novo Assembly and Characterization of Oryza officinalis Leaf Transcriptome by Using RNA-Seq. Biomed Res Int. 2015;2015:982065.

72. Lu X, Kim H, Zhong S, Chen H, Hu Z, Zhou B. De novo transcriptome assembly for rudimentary leaves in Litchi chinesis Sonn. and identification of differentially expressed genes in response to reactive oxygen species. BMC Genomics. 2014;15(1):805.

73. Ranjan A, Ichihashi Y, Farhi M, Zumstein K, Townsley B, David-Schwartz R, et al. De novo assembly and characterization of the transcriptome of the parasitic weed dodder identifies genes associated with plant parasitism. Plant Physiol. 2014;166(3):1186-99.

74. Laurent GS, Wahlestedt C, Kapranov P. The Landscape of long noncoding RNA classification. Trends Genet. 2015;31(5):239-51.

75. The IncRNA (Long Noncoding RNA) database. [http://www.Incrnadb.org/]. Accessed 20 September 2015.

76. Liu J, Jung C, Xu J, Wang H, Deng S, Bernad L, et al. Genome-wide analysis uncovers regulation of long intergenic noncoding RNAs in Arabidopsis. Plant Cell. 2012;24(11):4333-45.

77. Liu J, Wang H, Chua N. Long noncoding RNA transcriptome of plants. Plant Biotech J. 2015;13(3):319-28.

78. Webb EC. Enzyme nomenclature 1992. Recommendations of the Nomenclature Committee of the International Union of Biochemistry and Molecular Biology on the Nomenclature and Classification of Enzymes. Academic Press. 1992

79. Niederhuth CE, Patharkar OR, Walker JC. Transcriptional profiling of the Arabidopsis abscission mutant hae hsl2 by RNA-Seq. BMC Genomics. 2013;14:37.

80. Kanehisa M, Goto S. KEGG: kyoto encyclopedia of genes and genomes. Nucleic Acids Res. 2000;28(1):27-30

81. Iseli C, Jongeneel CV, Bucher P. ESTScan: a program for detecting, evaluating, and reconstructing potential coding regions in EST sequences. Int Conf Intell Syst Mol Bio. 1999;7:138-48.

82. Mott R. EST_GENOME: a program to align spliced DNA sequences to unspliced genomic DNA. Comput Appl Biosci. 1997;13:477-8.

83. Kumar S, Banks TW, Cloutier S. SNP discovery through next-generation sequencing and its applications. Int J Plant Genomics. 2012. doi:10.1155/ 2012/831460.
84. Zalapa JE, Cuevas H, Zhu H, Steffan S, Senalik D, Zeldin E, et al. Using nextgeneration sequencing approaches to isolate simple sequence repeat (SSR) loci in the plant sciences. Am J Bot. 2012;99(2):193-208.

85. Ramos AM, Crooijmans RP, Affara NA, Amaral AJ, Archibald AL, Beever JE, et al. Design of a high density SNP genotyping assay in the pig using SNPS identified and characterized by next generation sequencing technology. PLoS One. 2009;4(8):e6524.

86. Robbins MD, Sim SC, Yang W, Van Deynze A, van der Knaap E, Joobeur T, et al. Mapping and linkage disequilibrium analysis with a genome-wide collection of SNPs that detect polymorphism in cultivated tomato. J Exp Bot. 2011;62(6):1831-45.

87. Sim S, Durstewitz G, Plieske J, Wieseke R, Ganal MW, Van Deynze A, et al Development of a large SNP genotyping array and generation of highdensity genetic maps in tomato. PLoS One. 2012;7:e40563.

88. Garrison E, Marth G. Haplotype-based variant detection from short-read sequencing. arXiv preprint. arXiv. 2012;1207:3907.

89. Wittwer CT, Reed GH, Gundry CN, Vandersteen JG, Pryor RJ. High-resolution genotyping by amplicon melting analysis using LCGreen. Clin Chem. 2003;49(6):853-60

90. Daunay M. Eggplant. In: Prohens J, Nuez F, editors. Vegetables II: Handbook of plant Breeding. New York: Springer; 2008. p. 163-220.

91. Amar MH, Biswas MK, Zhang Z, Guo W. Exploitation of SSR, SRAP and CAPSSNP markers for genetic diversity of Citrus germplasm collection. Sci Hortic. 2011;128(3):220-7.

92. Pariasca-Tanaka J, Lorieux M, He C, McCouch S, Thomson MJ, Wissuwa M. Development of a SNP genotyping panel for detecting polymorphisms in Oryza glaberrima/O. sativa interspecific crosses. Euphytica. 2015;201(1):67-78.

93. Fan J, Chee MS, Gunderson KL. Highly parallel genomic assays. Nat Rev Genet. 2006;7(8):632-44.

94. Gupta P, Rustgi S, Mir R. Array-based high-throughput DNA markers for crop improvement. Heredity. 2008;101(1):5-18.

95. Abajian C. Sputnik. University of Washington Department of Molecular Biotechnology. 1994.

96. Ding Q, Li J, Wang F, Zhang Y, Li H, Zhang J, Gao J. Characterization and Development of EST-SSRs by Deep Transcriptome Sequencing in Chinese Cabbage (Brassica rapa L. ssp. pekinensis). Int J Genomics. 2015;2015:473028.

97. Chen H, Wang L, Wang S, Liu C, Blair MW, Cheng X. Transcriptome sequencing of mung bean (Vigna radiate L.) genes and the identification of EST-SSR markers. PLoS One. 2015;10(4):e0120273.

98. Zeng S, Xiao G, Guo J, Fei Z, Xu Y, Roe BA, et al. Development of a EST dataset and characterization of EST-SSRs in a traditional Chinese medicinal plant, Epimedium sagittatum (Sieb. Et Zucc.) Maxim. BMC Genomics. 2010;11(1):94.

99. Pinosio S, González-Martínez S, Bagnoli F, Cattonaro F, Grivet D, Marroni F, et al. First insights into the transcriptome and development of new genomic tools of a widespread circum-Mediterranean tree species, Pinus halepensis Mill. Mol Ecol Resour. 2014;14(4):846-56.

100. Gao C, Xin P, Cheng C, Tang Q, Chen P, Wang C, et al. Diversity Analysis in Cannabis sativa Based on Large-Scale Development of Expressed Sequence Tag-Derived Simple Sequence Repeat Markers. PLoS One. 2014;9(10):e110638.

101. Stàgel A, Portis $E$, Toppino L, Rotino GL, Lanteri S. Gene-based microsatellite development for mapping and phylogeny studies in eggplant. BMC Genomics. 2008;9(1):357.

102. Blanca J, Canizares J, Roig C, Ziarsolo P, Nuez F, Pico B. Transcriptome characterization and high throughput SSRs and SNPs discovery in Cucurbita pepo (Cucurbitaceae). BMC Genomics. 2011;12:104.

103. Schlautman B, Fajardo D, Bougie T, Wiesman E, Polashock J, Vorsa N, et al. Development and Validation of 697 Novel Polymorphic Genomic and ESTSSR Markers in the American Cranberry (Vaccinium macrocarpon Ait.). Molecules. 2015;20(2):2001-13.

104. Metzgar D, Bytof J, Wills C. Selection against frameshift mutations limits microsatellite expansion in coding DNA. Genome Res. 2000;10(1):72-80.

105. Li YC, Korol AB, Fahima T, Nevo E. Microsatellites within genes: structure, function, and evolution. Mol Biol Evol. 2004;21(6):991-1007.

106. Gonzalez-lbeas D, Blanca J, Roig C, Gonzalez-To M, Pico B, Truniger V, et al. MELOGEN: an EST database for melon functional genomics. BMC Genomics. 2007;8:306.

107. Durand J, Bodenes C, Chancerel E, Frigerio JM, Vendramin G, Sebastiani F, et al. A fast and cost-effective approach to develop and map EST-SSR markers: oak as a case study. BMC Genomics. 2010;11:570.

108. Zhou C, He X, Li F, Weng Q, Yu X, Wang Y, et al. Development of 240 novel EST-SSRs in Eucalyptus L'Hérit. Mol Breed. 2014;33(1):221-5. 
109. Kumar B, Kumar U, Yadav HK. Identification of EST-SSRs and molecular diversity analysis in Mentha piperita. The Crop Journal. 2015;3(4):335-42.

110. Varshney RK, Graner A, Sorrells ME. Genic microsatellite markers in plants: features and applications. Trends Biotechnol. 2005;23(1):48-55.

111. Poczai P, Varga I, Laos M, Cseh A, Bell N, Valkonen JP, et al. Advances in plant gene-targeted and functional markers: a review. Plant Methods. 2013;9(1):6.

112. The FastQC software. [http://www.bioinformatics.babraham.ac.uk/projects/ fastqc/]. Accessed 20 September 2015.

113. The NGS_CRUMBS software. [https://bioinf.comav.upv.es/ngs_crumbs/]. Accessed 20 September 2015.

114. Huang X, Madan A. CAP3: A DNA sequence assembly program. Genome Res. 1999;9(9):868-77.

115. Morgulis A, Gertz EM, Schäffer AA, Agarwala R. A fast and symmetric DUST implementation to mask low-complexity DNA sequences. J Comput Biology. 2006;13(5):1028-40.

116. The GOterm database. [http://geneontology.org/]. Accessed 20 September 2015.

117. Trapnell C, Pachter L, Salzberg SL. TopHat: discovering splice junctions with RNA-Seq. Bioinformatics. 2009;25(9):1105-11.

118. McKenna A, Hanna M, Banks E, Sivachenko A, Cibulskis K, Kernytsky A, et al. The Genome Analysis Toolkit: a MapReduce framework for analyzing nextgeneration DNA sequencing data. Genome Res. 2010;20(9):1297-303.

119. The eggplant genome database. [http://eggplant.kazusa.or.jp/]. Accessed 20 September 2015

120. The IGV software. [http://www.broadinstitute.org/igv/]. Accessed 20 September 2015.

121. The Primer3 software. [http://bioinfo.ut.ee/primer3-0.4.0/]. Accessed 20 September 2015.

122. Quinlan AR, Hall IM. BEDTools: a flexible suite of utilities for comparing genomic features. Bioinformatics. 2010;26(6):841-2.

\section{Submit your next manuscript to BioMed Central and we will help you at every step:}

- We accept pre-submission inquiries

- Our selector tool helps you to find the most relevant journal

- We provide round the clock customer support

- Convenient online submission

- Thorough peer review

- Inclusion in PubMed and all major indexing services

- Maximum visibility for your research

Submit your manuscript at www.biomedcentral.com/submit 